\title{
PREFERRED SETS IN TOPOLOGICAL DYNAMICS
}

\author{
SABER ELAYDI AND PING-FUN LAM
}

\begin{abstract}
The second author introduced a set of dynamical invariants called indivisibilities in one of his previous papers [3]. Following the same idea but from a different point of view this note concerns a class of invariants in transformation groups called the 'preferred sets'. For a special class of transformation groups, the number of permissible preferred sets is determined.
\end{abstract}

In Lam [3] a topological dynamical invariant $\left(\xi, Y, \mathcal{Q},\left\{K_{i}\right\}\right)$ called indivisibility was studied. The symbols represent a transformation group $\xi=(X, T)$, a subset $Y$ of $X$, the set of nets $\mathcal{Q}$ in the acting group $T$ and a family $\left\{K_{i}\right\}$ of subsets $X$. It is defined as follows: For every $K \in\left\{K_{i}\right\}$, whenever there exists a point $y_{0} \in Y$ such that for some $\alpha \in \mathcal{2}$ the net $y_{0} \alpha$ has a limit point in $K$, then the net $y \alpha$ has a limit point in $K$ for all $y \in Y$. In this paper we will consider the case where the set $\left\{K_{i}\right\}$ consists of only one set $K$. If $K$ is nonempty, we call it a preferred set of $Y$. We will study the family of preferred sets of $Y$ especially for the case that $Y$ has a finite number of preferred sets. In this paper we will denote a transformation group by $(X, T)$. The phase space $X$ will be a $T_{2}$ uniform space with a prescribed uniformity. The group $T$ is, in general, an arbitrary topological group (cf. [1],[2] for references).

Definition. A subset $K$ of $X$ is said to be a preferred set of $Y$ in $X$ if $K$ is nonempty and if there exists $y_{0} \in Y$ such that $y_{0} \alpha$ has a limit point in $K$ for a certain net $\alpha$, then $y \alpha$ has a limit point in $K$ for all $y \in Y$. If $K$ does not contain any proper subset which is a preferred set of $Y$, then $K$ is said to be a minimal preferred set (of $Y$ ).

If $X$ is compact, we let $E$ (not to be confused with $E(X)$, the set of all equicontinuous points of $X$ ) be the enveloping semigroup of $X$ (see [1]). Then a nonempty set $K \subset X$ is a preferred set of $Y \subset X$ if and only if for every $f \in E$ either $f(Y) \cap K=\varnothing$ or $f(Y) \subset K$. If $X$ is noncompact, a set $Y$ need not have a preferred set. In fact if $Y$ is the set $E(X)$ of Example 5.4 of Lam [3], and if the fixed point $\left(\frac{1}{2}, 0,0\right)$ is removed from the phase space $X$, then the set $E(X)$ has no preferred sets. We begin with some lemmas.

Lemma 1. Let $K$ and $Y$ be subsets of $X$. The following property holds. If $K$ is a preferred set of $Y$ which intersects $Y$, then $K$ contains $Y$.

Received by the editors June 28, 1976 and, in revised form, September 29, 1976. AMS (MOS) subject classifications (1970). Primary 54H20. 
Proof. We need only take $\alpha$ to be a net of identity elements in the definition of a preferred set.

The notion of preferred sets may be used to characterize the property that a set is 23-indivisible studied in [3,3.1] (in notation $2 H Y$ ). The third indivisibility was considered in [3] as an important property. It is now characterized by a few other properties, among them the following is one.

Definition. A set $Y \subset X$ is said to be totally equitable if for every decomposition of $X$ if all but one of the sets are preferred sets of $Y$, then the last is also a preferred set of $Y$. Equivalently, $Y$ is totally equitable if for every preferred set $K$ of $Y$ which is not $X$, its complement $X-K$ is also a preferred set of $Y$. For a subset $Y$ of $X$ the following properties hold. If $\left\{K_{i}\right\}$ is a nonempty family of preferred sets of $Y$, then $\cup_{i} K_{i}$ is a preferred set of $Y$, and if $\bigcap_{i} K_{i} \neq \varnothing$ then the intersection is also a preferred set of $Y$.

LEMMA 2. If $K_{1}$ and $K_{2}$ are two distinct subsets of $X$ such that $K_{2} \subset K_{1}$ and if both sets are preferred sets of $Y$, then the difference set $K_{1}-K_{2}$ is also a preferred set of $Y$.

Proof. The proof is actually very simple. Suppose the set $K_{1}-K_{2}$ is not preferred. Then there exist two points $a, b \in Y$ and $\alpha \in \mathcal{Z}$ such that $a \alpha$ has a limit point in $K_{1}-K_{2}$ and $b \alpha$ does not have a limit point in $K_{1}-K_{2}$. We may assume that $x \alpha$ converges to a point in a compactification of $X$, for instance, the one-point compactification of [3], for all $x \in Y$. Now $K_{1}$ is a preferred set of $Y$. Since $\lim a \alpha$ is in $K_{1}-K_{2}$, we must also have that $\lim b \alpha \in K_{1}$. But we also have $\lim b \alpha \notin K_{1}-K_{2}$, hence $\lim b \alpha \in K_{2}$. Since $K_{2}$ is a preferred set of $Y, \lim a \alpha \in K_{2}$, which contradicts that $\lim a \alpha \in K_{1}$ $-K_{2}$. Hence $K_{1}-K_{2}$ is a preferred set of $Y$.

Lemma 3. For $Y \subset X$ the following statements are pairwise equivalent.

(1) $2 H+Y$, i.e. $X$ is a preferred set of $Y$.

(2) $Y$ is totally equitable and it has at least one preferred set.

(3) The family of preferred sets of $Y$ forms a covering of $X$.

(4) $X$ is decomposed into a disjoint union of minimal preferred sets of $Y$.

Proof. (1) $\Rightarrow$ (2). Suppose $Y$ has a preferred set $K$ such that $X-K \neq \varnothing$. According to Lemma 2 the set $X-K$ is a preferred set of $Y$. Hence $Y$ is totally equitable.

$(2) \Rightarrow(3)$. Let $\left\{K_{i}\right\}$ be the nonempty family of all preferred sets of $Y$. The $\cup_{i} K_{i}$ is then a preferred set of $Y$. Since $Y$ is totally equitable and $X-\cup_{i} K_{i}$ cannot be a preferred set, we have $X=\cup_{i} K_{i}$

(3) $\Rightarrow(4)$. Each $x \in X$ is contained in a preferred set, hence $M=\cap\{K \mid K$ a preferred set of $Y$ contains $x\}$ is a preferred set. If $M$ is not a minimal preferred set of $Y$, then there would be a preferred proper subset $K_{0}$ in $M$. Since $M-K_{0}$ is also a preferred set of $Y, M$ is then either equal to $K_{0}$ or equal to $M-K_{0}$ which is a contradiction.

$(4) \Rightarrow(1)$. Obvious. The proof of Lemma 3 is completed. 
As a by-product of considering Lemma 3, we have the following simple result.

THEOREM 1. If the number of preferred sets of $Y \subset X$ is finite, then it is $2^{n}-1$, where $n=0,1,2, \ldots$.

Proof. Either $Y$ has no preferred set or there exists a maximum preferred set $R$, which contains all other preferred sets. The proof of Lemma 3 shows that $R$ is decomposed into a disjoint union of minimal preferred sets of $Y$. It follows easily that the number of preferred sets is $2^{n}-1$. Easy examples show that all numbers $2^{n}-1$ can be attained.

We now consider three theorems, which are the main results of the paper. The theorems can be applied to subsets $Y$ of $E(X)$.

TheOREM 2. For a transformation group $(X, T)$, where $X$ is a metric space which is locally compact and connected and $T$ is also connected, let $N(X)$, the set of all nonequicontinuous points of $X$, be 0-dimensional. Then the number of preferred sets of $E(X)$ is either $0,1,3$ or 7 .

Proof. Since $T$ is connected, the components of $E(X)$ are invariant. We take one of them and call it $M$. Let $F=\mathrm{Cl}(M)$; then $F$ is closed and invariant. We consider the restricted transformation group $(F, T)$ and denote by $E(F)$ its set of equicontinuous points. By Lemma 1 there exists at most one minimal preferred set of $E(X)$ overlapping $E(X)$. We claim that if $E(X)$ has a fixed point $x_{0}$, then $E(X)$ has at most one other minimal preferred set. Thus let $K$ be another preferred set of $E(X)$; then $K \subset N(X)$. Let $p \in K$. By a lemma proved in Lam [4] (see Lemma 4 of this paper) there exists $a \in E(X)$ with a sequence $\alpha$ in $T$ such that $\lim a \alpha=p$. We may assume that $\alpha$ has the property as that in the proof of Lemma 2 , then we may define a function $f: E(X) \rightarrow X^{*}, f(x)=\lim x \alpha$, where $X^{*}$ is the one-point compactification of $X$. Since $K$ is a preferred set of $E(X)$, we have $f(M) \subset K$. It is clear that $f$ is continuous and therefore $f(M)$ is a singleton, say $q$ in the totally disconnected set $K$. Since $x_{0} \in E(F)$, we have lim $x_{0} \alpha=q$. However, $x_{0}$ is a fixed point, hence $x_{0}=q$. Hence $x_{0} \in K$. There is then at most one such set $K$. Hence $E(X)$ has 1 or 3 preferred sets in case there is a minimal preferred set. It remains to show the case when $E(F)$ has no fixed points. Since $T$ is connected, the set $N(X)$ under the present assumption, consists of fixed points and since the set of fixed points is closed, we may assume $\mathrm{Cl}(N(X)) \cap$ $E(F)=\varnothing$. If $N(F)$ is the set of nonequicontinuous points of $(F, T)$, then

$$
\mathrm{Cl}(N(X)) \cap F \subset N(F) \subset \mathrm{Cl}(N(X)) \cap F .
$$

Hence

$$
N(F)=N(X) \cap F=\mathrm{Cl}(N(X)) \cap F .
$$

In particular, $N(F)$ is closed, and if it is not empty it is what is called a strictly almost equicontinuous transformation group (SAE) in Lam [4]. By definition a transformation group $(X, T)$ is SAE if $X$ is locally compact 
metric, $N(X)$ is 0-dimensional, $\mathrm{Cl}(p T)$ is compact if $p \in N(X)$ and $E(X)$ is completely indivisible by 2 (by that we mean $(\xi, E(X), \mathcal{Q}, \mathcal{K})$ are indivisibilities for (i) $\mathcal{K}$ to be the set of components of $N(X)$ and for (ii) $\mathcal{K}$ to be the single set $X$.) It was proved there that if $E(X)$ is connected and $N(X)$ is closed then the indivisibility conditions were satisfied. Theorem 1.17 in [4] states that for SAE the set $N(F)$ consists of none, one or two points. If $K$ is a minimal preferred set of $E(X)$ and $p$ is a point in it we can define a function $f: E(X) \rightarrow X^{*}$ and show that $p \in f(M) \subset K$. Since $M$ is a subset of $F$ which is closed and invariant we have $f(M) \subset F$. Hence $f(M) \subset K \cap F \subset N(X)$ $\cap F=N(F)$. It follows that there can be at most two such sets $K$. It should be noted that under the hypothesis of the theorem, the set $N(X)$ can be infinite (see [3, Example 5.4]).

Lemma 4. Let $X$ be a locally compact and connected metric space. Let $N(X)$ be nonempty, 0-dimensional and if $p \in N(X)$, then $\mathrm{Cl}(p T)$ is compact. Then for every 0-dimensional set $A \subset X$ any sequence $\left\{t_{i}\right\}$ which is nonequicontinuous at a point $p \in N(X)$ has a subsequence $\left\{t_{i_{j}}\right\}$ of $\left\{t_{i}\right\}$ and a point $q \in E(X)-A$ such that $\lim q t_{i_{j}}^{-1}=p$.

Proof. The case when $A=\varnothing$ is proved in Lemma 1.11 of [4]. A slight modification of the proof is sufficient for the present case.

THEOREM 3. Let $X$ again be a locally compact connected metric space, $N(X)$ 0 -dimensional and $\mathrm{Cl}(p T)$ compact for all $p \in N(X)$. If $T$ is abelian and $E(X)$ has an invariant component, then the number of preferred sets of $E(X)$ is $0,1,3$ or 7 .

Proof. Let $R$ in Theorem 1 exist and let $M$ be the invariant component of $E(X)$. Consider $F=\mathrm{Cl}(M)$. Let $K_{1}$ be a minimal preferred set of $E(X)$ which overlaps $E(X)$; in general $K_{1}$ exists. If there exists a minimal preferred set $K_{2}$ of $E(X)$ not intersecting $E(X)$, let $p \in K_{2}$. Then there exists a sequence $\alpha$ in $T$ such that $\lim a \alpha=p$ for some $a \in E(X)$. We may assume that $f$ : $E(X) \rightarrow X^{*}$, given by $f(y)=\lim y \alpha$, exists. Then $f(M)$ is a singleton $q \in K_{2}$, and $q \in R \cap(N(X) \cap F)$. If we can show that there are at most two points in $B=R \cap(N(X) \cap F)-K_{1}$, then there can be at most two such subsets $K_{2}$ of $X$.

Thus let there be three distinct points $p_{1}, p_{2}$ and $p_{3}$ in $B$. We first show that they are fixed points. Since the preferred sets of $E(X)$ cover $B$ we may assume that $p_{1} \in K_{2}$. Then $\lim x \alpha=p_{1}$ for all $x \in M_{1}=E(X) \cap F$. The set $M_{1}$ is invariant and connected. Let $x_{1} \in M_{1}$ and $t \in T$, then $x_{1} t \in M_{1}$. Since $T$ is abelian, we have

$$
p_{1} t=\lim x_{1} \alpha t=\lim x_{1} t \alpha=p_{1} .
$$

Hence, $p_{1}$ and, similarly, $p_{2}$ and $p_{3}$ are fixed points. Since $M_{1}$ is dense in $F$, in particular, at $p_{2}$ and $p_{3}$ in $(F, T)$, we see that the sequence $\alpha$ is nonequicontinuous at the fixed points $p_{2}$ and $p_{3}$. Let $A$ in Lemma 4 be $E(F)-M_{1}$. By applying Lemma 4 twice on $(F, T)$ we can find two points $a, b \in E(F)-A$ 
$=M_{1}$ and a sequence $\left\{t_{i_{j}}\right\}$ of $\left\{t_{i}\right\}$ such that $\lim a t_{i_{j}}^{-1}=p_{2}$ and $\lim b t_{i_{j}}^{-1}=p_{3}$. However, it is easily seen that each of the singleton sets $\left\{p_{2}\right\}$ and $\left\{p_{3}\right\}$ is a preferred set of $M_{1}$. We then have a contradiction. Hence $B$ has at most two points.

LEMMA 5. Let $A$ be a syndetic subgroup of $T$ (i.e. there exists a compact set $K \subset T$ for the subgroup $T$ such that $A K=T)$. If $\mathrm{Cl}(p T)$ is compact for all $p \in N(X)$, then $N(X)$ is also the set of all nonequicontinuous points of $A$.

Proof. Straightforward argument.

We now state the theorem for the last case.

TheOREM 4. Let $X$ be a locally compact and connected metric space and let $N(X)$ be finite. Then the number of preferred sets of $E(X)$ is $0,1,3$ or 7 .

Proof. We may assume that $E(X)$ has at least one preferred set $K$. If $K \cap E(X) \neq \varnothing$, then by Lemma $1, E(X) \subset K$. If so, then $K$ may be assumed to be the minimal preferred set containing $E(X)$. Let $K_{1}$ be any other possible minimal preferred set of $E(X)$. Then $K_{1} \subset N(X)$. Let $p \in K_{1}$. By Lam [3, Lemma 1.11] there exists a sequence $\alpha \in \mathcal{2}$ and $\lim x \alpha \in K_{1}$ for all $x \in E(X)$. Consider the syndetic subgroup $A=\{t \in T \mid x t=x$ for all $x \in N(X)\}$ and the transformation group $(X, A)$. By Lemma 5 the set of nonequicontinuous points remains unchanged. Note that under $A$ the set of $N(X)$ consists of fixed points. Now since $E(X)$ is dense in $X$ by a similar argument as given in the proof of Theorem 3, we see that if $K_{2}$ and $K_{3}$ are two other minimal preferred sets contained in $N(X)$, then $K_{2}$ and $K_{3}$ consist of nonequicontinuous points of $\alpha$. The same argument applies to show that $K_{2}=K_{3}$. Hence $E(X)$ has at most 3 minimal preferred sets and the argument is completed.

\section{REFERENCES}

1. Robert Ellis, Lectures on topological dynamics, Benjamin, New York, 1969. MR 42 \#2463.

2. W. H. Gottschalk and G. A. Hedlund, Topological dynamics, Amer. Math. Soc. Colloq. Publ., vol. 36, Amer. Math. Soc., Providence, R.I., 1955. MR 17, 650.

3. Ping-Fun Lam, Equicontinuity and indivisibility in transformation groups, Trans. Amer. Math. Soc. 174 (1972), 399-424. MR 46 \#8201.

4. , Almost equicontinuous transformation groups, Trans. Amer. Math. Soc. 195 (1974), 165-200. MR 51 \#14126.

Department of Mathematics, University of Missouri, Columbia, Missouri 65201 